Homology, Homotopy and Applications, vol.18(2), 2016, pp.345-357

\title{
HOMOTOPY THEORY OF COFIBRATION CATEGORIES
}

\author{
KAROL SZUMIŁO \\ (communicated by Emily Riehl)
}

\begin{abstract}
We construct a fibration category of cofibration categories which constitutes a convenient framework for the homotopy theory of cofibration categories.
\end{abstract}

\section{Introduction}

Categories of fibrant objects were introduced by Brown [Bro73] as a more flexible alternative to Quillen's model categories. In this paper we will consider fibration categories, a slight variation of Brown's notion, and their duals cofibration categories. Our main result is the following (Theorem 2.8).

Theorem. The category of (small) cofibration categories carries a structure of a fibration category.

In a manner similar to a model category, a cofibration category presents a homotopy theory and provides structure allowing for construction of homotopy colimits. Cofibration and fibration categories never became nearly as popular as model categories, but a number of contributions has been made by, among the others, Anderson [And78], Baues [Bau89, Bau99], Cisinski [Cis10a] and Rădulescu-Banu [RB06]. Moreover, Waldhausen [Wal85] introduced a closely related notion of a category with cofibrations and weak equivalences (nowadays usually called a Waldhausen category) for the purpose of developing a general framework for algebraic K-theory. Subsequently, a close connection to abstract homotopy theory was made by Cisinski [Cis10b].

The motivation behind cofibration and fibration categories is two-fold. Firstly, they can be used to formalize certain homotopy theories that do not fit into the framework of model categories. Examples include the fibration category of $C^{*}$-algebras [AG97] and categorical models of homotopy type theory [AKL15, Theorem 3.2.5]. A few more examples are briefly discussed in [Szu14a, Section 1.4]. Secondly, the structure of a cofibration category is less rigid than that of a model category which makes it possible to carry out certain constructions that might be impossible in the context of model categories, e.g., the category of diagrams in a cofibration category over a fixed indexing category always carries a structure of cofibration category itself [RB06, Theorem 9.5.5(1)].

Received July 14, 2015, revised May 8, 2016; published on November 18, 2016.

2010 Mathematics Subject Classification: 55U35 (Primary), 18G55 (Secondary).

Key words and phrases: homotopy theory, cofibration category.

Article available at http://dx.doi.org/10.4310/HHA.2016.v18.n2.a19

Copyright (C) 2016, International Press. Permission to copy for private use granted. 
In the present paper we take advantage of the flexibility of cofibration categories to provide efficient tools for working with the homotopy theory of cofibration categories. In Section 1 we introduce cofibration categories and briefly revisit the well known techniques of homotopical algebra that will be needed later. In Section 2 we define fibrations of cofibration categories and prove our main theorem, i.e., that the category of (small) cofibration categories is a fibration category.

This paper is the first in the series of three that summarize the results of the author's thesis [Szu14a]. (See also [Szu14b] for a slightly edited version.) The second paper [Szu16] introduces a new construction of a cocomplete quasicategory associated with a cofibration category called the quasicategory of frames. The third one [Szu] shows that it is possible to construct a cofibration category from a cocomplete quasicategory in a way that establishes an equivalence between the homotopy theory of cofibration categories and the homotopy theory of cocomplete quasicategories.

\section{Acknowledgments}

This paper is based on a part of my thesis [Szu14a] which was written while I was a doctoral student in Bonn International Graduate School in Mathematics and, more specifically, Graduiertenkolleg 1150 "Homotopy and Cohomology" and International Max Planck Research School for Moduli Spaces. I want to thank everyone involved for creating an excellent working environment.

I want to thank Clark Barwick, Bill Dwyer, André Joyal, Chris Kapulkin, Lennart Meier, Thomas Nikolaus, Chris Schommer-Pries, Peter Teichner and Marek Zawadowski for conversations on various topics which were very beneficial to my research.

I am especially grateful to Viktoriya Ozornova and Irakli Patchkoria for reading an early draft of my thesis. Their feedback helped me make many improvements and avoid numerous errors.

Above all, I want to express my gratitude to my supervisor Stefan Schwede whose expertise was always invaluable and without whose support this thesis could not have been written.

\section{Definitions and basic properties}

Definitions of (co)fibration categories found throughout the literature vary in details. The one stated here is almost the same as (the dual of) Brown's original definition [Bro73, p. 420]. Since we use [RB06] as our main source we point out that in the terminology of this paper the definition below corresponds to "precofibration categories with all objects cofibrant and the 2-out-of- 6 property". It should be noted that the only (but essential) use of the 2-out-of-6 property in the present paper takes place in the proof of Proposition 2.2. Comparisons to other definitions can be found in [RB06, Chapter 2]. We do not commit much space to the discussion of basic properties of cofibration categories, we refer the reader to [RB06] for these.

Definition 1.1. A cofibration category is a category $\mathcal{C}$ equipped with two subcategories: the subcategory of weak equivalences (denoted by $\stackrel{\sim}{\rightarrow}$ ) and the subcategory of cofibrations (denoted by $\longmapsto$ ) such that the following axioms are satisfied. (Here, an acyclic cofibration is a morphism that is both a weak equivalence and a cofibration.) 
(C0) Weak equivalences satisfy the 2-out-of-6 property, i.e., if

$$
W \stackrel{f}{\longrightarrow} X \stackrel{g}{\longrightarrow} Y \stackrel{h}{\longrightarrow} Z
$$

are morphisms of $\mathcal{C}$ such that both $g f$ and $h g$ are weak equivalences, then so are $f, g$ and $h$ (and thus also $h g f$ ).

(C1) Every isomorphism of $\mathcal{C}$ is an acyclic cofibration.

(C2) An initial object exists in $\mathcal{C}$.

(C3) Every object $X$ of $\mathcal{C}$ is cofibrant, i.e., if 0 is the initial object of $\mathcal{C}$, then the unique morphism $0 \rightarrow X$ is a cofibration.

(C4) Cofibrations are stable under pushouts along arbitrary morphisms of $\mathcal{C}$ (in particular, these pushouts exist in $\mathcal{C}$ ). Acyclic cofibrations are stable under pushouts along arbitrary morphisms of $\mathcal{C}$.

(C5) Every morphism of $\mathcal{C}$ factors as a composite of a cofibration followed by a weak equivalence.

Cofibration categories are, in a sense, more general than model categories. Namely, if $\mathcal{M}$ is a model category, then its full subcategory of cofibrant objects $\mathcal{M}_{\text {cof }}$ with weak equivalences and cofibrations inherited from $\mathcal{M}$ satisfies the above axioms. There are examples of (co)fibration categories that do not come from model categories. Some of those are presented in [Szu14a, Section 1.4].

Next, we introduce morphisms of cofibration categories and recall a standard method of verifying homotopy invariance of functors between cofibration categories.

Definition 1.2. A functor $F: \mathcal{C} \rightarrow \mathcal{D}$ between cofibration categories is exact if it preserves cofibrations, acyclic cofibrations, initial objects and pushouts along cofibrations.

Lemma 1.3 (Brown's Lemma). If a functor between cofibration categories sends acyclic cofibrations to weak equivalences, then it preserves all weak equivalences. In particular, exact functors preserve weak equivalences.

Proof. The proof of [Hov99, Lemma 1.1.12] works for cofibration categories. (See also the proof of [Bro73, Lemma 4.1] where this result first appeared.)

The axioms formulated above describe finitely (homotopy) cocomplete cofibration categories. The category of such (small) cofibration categories with exact functors as morphisms will be denoted by CofCat.

We can use cofibration categories to describe homotopy theories with arbitrary homotopy colimits using the following additional axioms.

(C6) Cofibrations are stable under sequential colimits, i.e., given a sequence of cofibrations

$$
A_{0} \longmapsto A_{1} \longmapsto A_{2} \longmapsto \cdots,
$$

its colimit $A_{\infty}$ exists and the induced morphism $A_{0} \rightarrow A_{\infty}$ is a cofibration. Acyclic cofibrations are stable under sequential colimits. This axiom is often stated as "(acyclic) cofibrations are closed under transfinite composition of length $\omega "$. 
$(\mathrm{C} 7-\kappa)$ Coproducts of $\kappa$-small families of objects exist. Cofibrations and acyclic cofibrations are stable under $\kappa$-small coproducts.

Axiom (C7) is parametrized by a regular cardinal number $\kappa$. A set is $\kappa$-small if it has fewer than $\kappa$ elements and a $\kappa$-small coproduct is one indexed over a $\kappa$-small set. We say that a cofibration category is (homotopy) $\kappa$-cocomplete for $\kappa>\aleph_{0}$ if it satisfies (C6) and $(\mathrm{C} 7-\kappa)$. For $\kappa=\aleph_{0}$ the name (homotopy) $\aleph_{0}$-cocomplete cofibration category will refer to a cofibration category satisfying the original axioms $(\mathrm{C} 0-5)$. We will write CofCat ${ }_{\kappa}$ to denote the category of such (small) cofibration categories where morphisms are $\kappa$-cocontinuous functors, i.e., exact functors that preserve colimits of sequences of cofibrations (for $\kappa>\aleph_{0}$ ) and $\kappa$-small coproducts.

We will say that a cofibration category is (homotopy) cocomplete if it satisfies (C6) and $(\mathrm{C} 7-\kappa)$ for all $\kappa$.

The results of the remaining sections apply to CofCat ${ }_{\kappa}$ for arbitrary $\kappa$ but we will give proofs only for $\kappa=\aleph_{0}$ since arguments involving axioms (C6) and (C7) are straightforward analogues of the ones involving $(\mathrm{C} 4)$.

\section{Fibration category of cofibration categories}

We are now ready to introduce the homotopy theory of cofibration categories. For this it is sufficient to define a class of weak equivalences in the category of cofibration categories which is what we will do next. Later, we will proceed to define fibrations of cofibration categories and prove that they satisfy the axioms of a fibration category.

Definition 2.1. An exact functor $F: \mathcal{C} \rightarrow \mathcal{D}$ is a weak equivalence if it induces an equivalence $\mathrm{Ho} \mathcal{C} \rightarrow \mathrm{Ho} \mathcal{D}$.

This notion is closely related to the Waldhausen approximation properties first formulated by Waldhausen as criteria for an exact functor to induce an equivalence of the algebraic K-theory spaces [Wal85, Section 1.6]. Later, Cisinski showed that an exact functor satisfies (slightly reformulated) Waldhausen approximation properties if and only if it is a weak equivalence in the sense of the definition above. This is made precise in the following proposition.

Proposition 2.2 ([Cis10a, Théorème 3.19]). An exact functor $F: \mathcal{C} \rightarrow \mathcal{D}$ is a weak equivalence if and only if it satisfies the following properties.

(App1) $F$ reflects weak equivalences.

(App2) Given a morphism $f: F A \rightarrow Y$ in $\mathcal{D}$, there exists a morphism $i: A \rightarrow B$ in $\mathcal{C}$ and a commutative diagram

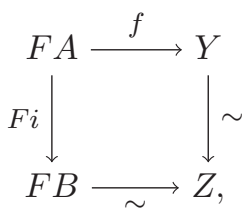

in $\mathcal{D}$.

We are now ready to define fibrations of cofibration categories. 
Definition 2.3. Let $P: \mathcal{E} \rightarrow \mathcal{D}$ be an exact functor of cofibration categories.

(1) $P$ is an isofibration if for every object $A \in \mathcal{E}$ and an isomorphism $g: P A \rightarrow Y$ there is an isomorphism $f: A \rightarrow B$ such that $P f=g$.

(2) It is said to satisfy the lifting property for factorizations if for any morphism $f: A \rightarrow B$ of $\mathcal{E}$ and a factorization

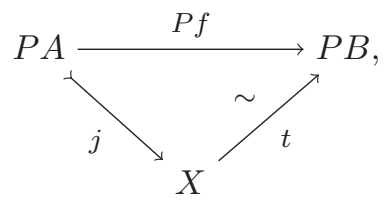

there exists a factorization

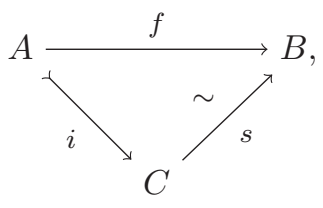

such that $P i=j$ and $P s=t$ (in particular, $P C=X$ ).

(3) It has the lifting property for pseudofactorizations if for any morphism $f: A \rightarrow$ $B$ of $\mathcal{E}$ and a diagram

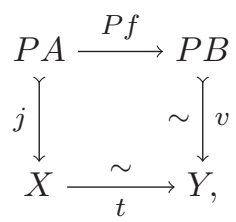

there exists a diagram

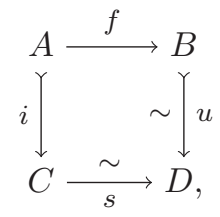

such that $P i=j, P s=t$ and $P u=v$ (in particular, $P C=X$ and $P D=Y$ ).

(4) We say that $P$ is a fibration if it is an isofibration and satisfies the lifting properties for factorizations and pseudofactorizations.

The first two conditions are motivated by Proposition 2.4 below which says that pullbacks along fibrations exist in the category of cofibration categories. The isofibration property is used to show that the categorical pullback has all required colimits while the lifting properties for factorizations is used to show that it has factorizations. The lifting property for pseudofactorizations is more technical and is only used in Proposition 2.5 which implies that acyclic fibrations are stable under pullback. Informally, a pseudofactorization is a "factorization into a cofibration and a zig-zag of weak equivalences". The need for such a notion arises because some morphisms of the homotopy category of a cofibration category cannot be represented by single morphisms of the underlying category. 
While the notion of a fibration between cofibration categories seems abstract, such functors are abundant. In [Szu16, Section 1] we present a number of examples and methods of constructing fibrations.

This definition can be restated in a more technical but convenient way. We define a category $\overline{\text { CofCat }}$ containing the category of cofibration categories CofCat as a non-full subcategory. Objects of CofCat are small categories equipped with two subcategories: the subcategory of weak equivalences and the subcategory of cofibrations such that all identity morphisms are acyclic cofibrations. Morphisms are functors that preserve both weak equivalences and cofibrations.

An exact functor between cofibration categories is a fibration if and only if it has

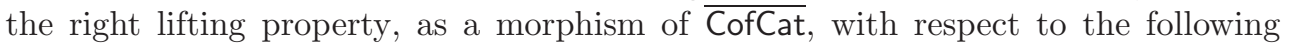
functors.

- The inclusion of [0] into $E(1)$ (the groupoid freely generated by an isomorphism $0 \rightarrow 1)$.

- The inclusion of [1] (with only identities as weak equivalences or cofibrations) into

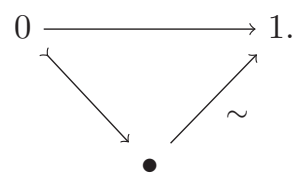

- The inclusion of $[1] \times[0]$ (with only identities as weak equivalences or cofibrations) into

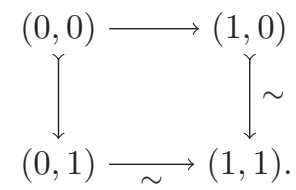

Proposition 2.4. Let $F: \mathcal{C} \rightarrow \mathcal{D}$ and $P: \mathcal{E} \rightarrow \mathcal{D}$ be exact functors between cofibration categories with $P$ a fibration. Then a pullback of $P$ along $F$ exists in CofCat.

Proof. Form a pullback of $P$ along $F$ in the category of categories:

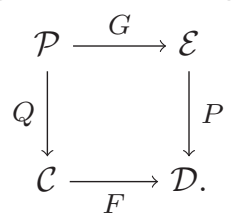

Define a morphism $f$ of $\mathcal{P}$ to be a weak equivalence (respectively, a cofibration) if both $G f$ and $Q f$ are weak equivalences (respectively, cofibrations). Then the above square becomes a pullback in CofCat.

Now we check that $\mathcal{P}$ is a cofibration category.

$(\mathrm{C} 0-1)$ In $\mathcal{P}$ weak equivalences satisfy 2 -out-of- 6 and all isomorphisms are acyclic cofibrations since this holds in both $\mathcal{C}$ and $\mathcal{E}$.

$(\mathrm{C} 2-3)$ Let $0_{\mathcal{C}}$ be an initial object of $\mathcal{C}$. Since $P$ is an isofibration there is an initial object $0_{\mathcal{E}}$ of $\mathcal{E}$ such that $P 0_{\mathcal{E}}=F 0_{\mathcal{C}}$. Then $\left(0_{\mathcal{C}}, 0_{\mathcal{E}}\right)$ is an initial object of $\mathcal{P}$. 
Moreover, every object of $\mathcal{P}$ is cofibrant since this holds in both $\mathcal{C}$ and $\mathcal{E}$.

(C4) Let $\ulcorner$ denote the poset of proper subsets of $\{0,1\}$ and let $X:\ulcorner\rightarrow \mathcal{P}$ be a span with $X_{\varnothing} \rightarrow X_{0}$ a cofibration. Let $S$ be a colimit of $Q X$ in $\mathcal{C}$, then $F S$ is a colimit of $F Q X=P G X$ in $\mathcal{D}$ since $F$ is exact. Since $P$ is an isofibration we can choose a colimit $T$ of $G X$ in $\mathcal{E}$ so that $P T=F S$. Then $(S, T)$ is a colimit of $X=(Q X, G X)$ in $\mathcal{P}$. Thus pushouts along cofibrations exist in $\mathcal{P}$ and both cofibrations and acyclic cofibrations are stable under pushouts since this holds in both $\mathcal{C}$ and $\mathcal{E}$.

(C5) Let $f: A \rightarrow B$ be a morphism of $\mathcal{P}$. Pick a factorization of $Q f$ as

$$
Q A \longmapsto C \stackrel{\sim}{\rightarrow} Q B
$$

in $\mathcal{C}$. Then $F Q f=P G f$ factors as

$$
P G A=F Q A \longmapsto F C \stackrel{\sim}{\rightarrow} F Q B=P G B
$$

and we can lift this factorization to a factorization of $G f$ as

$$
G A \longmapsto E \stackrel{\sim}{\rightarrow} G B .
$$

It follows that

$$
A=(Q A, G A) \longmapsto(C, E) \stackrel{\sim}{\rightarrow}(Q B, G B)=B
$$

is a factorization of $f$.

This completes the verification that $\mathcal{P}$ is a cofibration category.

It follows from the construction of $\mathcal{P}$ that $Q$ and $G$ are exact and that given a square

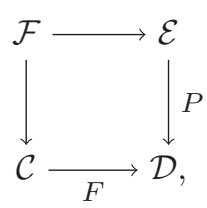

of cofibration categories and exact functors, the induced functor $\mathcal{F} \rightarrow \mathcal{P}$ is also exact. Hence the square we constructed is a pullback in the category of cofibration categories.

The next proposition will imply the stability of acyclic fibrations under pullbacks. It also serves as a useful criterion for verifying that an exact functor is a weak equivalence.

Proposition 2.5. An exact functor $P: \mathcal{C} \rightarrow \mathcal{D}$ is an acyclic fibration if and only if it is a fibration, satisfies (App1) and the right lifting property (in $\overline{\text { CofCat) with respect }}$ to the inclusion of [0] into

$$
0 \longmapsto 1 \text {. }
$$

Proof. First assume that $P$ satisfies the properties above. We need to check that it satisfies (App2). Let $f: P A \rightarrow Z$ be a morphism of $\mathcal{D}$. Factor $f$ as a composite of $j: P A \longmapsto Y$ and $Y \stackrel{\sim}{\rightarrow} Z$ and apply the lifting property above to find a cofibration 
$i: A \longmapsto B$ such that $P i=j$. This yields a diagram

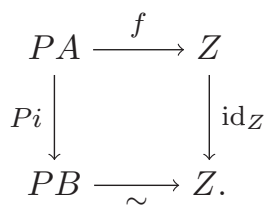

Conversely, assume that $P$ is an acyclic fibration. We need to check that it satisfies the lifting property above. Consider a cofibration $j: P A \longmapsto Y$ and apply (App2) to it to get $f: A \rightarrow B$ and a diagram

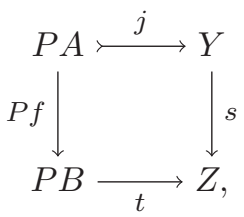

with both $s$ and $t$ weak equivalences. We factor $[t, s]: P B \amalg_{P A} Y \rightarrow Z$ as a composite of $\left[t^{\prime}, s^{\prime}\right]: P B \amalg_{P A} Y \longmapsto W$ and $W \stackrel{\sim}{\rightarrow} Z$. So we obtain the square on the left
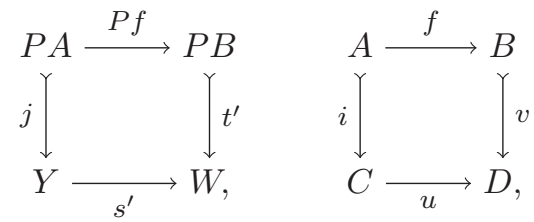

with both $s^{\prime}$ and $t^{\prime}$ weak equivalences. We can now apply the lifting property for pseudofactorizations to get the square on the left with $u$ and $v$ weak equivalences such that $P u=s^{\prime}, P v=t^{\prime}$ and (most importantly) $P i=j$.

Next, we proceed to the construction of factorizations. We will make use of homotopical categories which provide useful models of homotopy theories as discussed at length in [DHKS04]. However, we need them only as a technical tool to keep track of certain homotopical properties of diagrams in cofibration categories.

Definition 2.6. A homotopical category is a category equipped with a subcategory whose morphisms are called weak equivalences such that every identity morphism is a weak equivalence and the 2-out-of- 6 property holds.

A functor $I \rightarrow J$ between homotopical categories is homotopical if it preserves weak equivalences. In particular, for any cofibration category $\mathcal{C}$ and a homotopical category $J$ the homotopical functors $J \rightarrow \mathcal{C}$ will be called homotopical diagrams. The notation $\mathcal{C}^{J}$ will always refer to the category of all homotopical diagrams $J \rightarrow \mathcal{C}$. It is itself a homotopical category with levelwise weak equivalences. If $J$ is a plain category, then it will be considered as a homotopical category with the trivial homotopical structure, i.e., with only isomorphisms as weak equivalences. On the other hand, $\widehat{J}$ will denote $J$ equipped with the largest homotopical structure, i.e., the one where all morphisms are weak equivalences.

Let $\mathcal{C}$ be a cofibration category and let $\widehat{\lrcorner}$ denote the poset of non-empty subsets of $\{0,1\}$. Make it into a homotopical poset by declaring all morphisms to be weak equivalences. Call a diagram $X: \widehat{\lrcorner} \rightarrow \mathcal{C}$ cofibrant if both $X_{0} \rightarrow X_{01}$ and $X_{1} \rightarrow X_{01}$ are 
cofibrations in $\mathcal{C}$. Let $P \mathcal{C}$ denote the category of all homotopical cofibrant diagrams $\widehat{\lrcorner} \rightarrow \mathcal{C}$ (i.e., $X$ such that both $X_{0} \rightarrow X_{01}$ and $X_{1} \rightarrow X_{01}$ are acyclic cofibrations). Define weak equivalences in $P \mathcal{C}$ as levelwise weak equivalences and define a morphism $A \rightarrow X$ to be a cofibration if all

$$
\begin{aligned}
A_{0} & \rightarrow X_{0}, \\
A_{1} & \rightarrow X_{1}, \\
A_{01} \amalg_{A_{0}} X_{0} & \rightarrow X_{01} \text { and } \\
A_{01} \amalg_{A_{1}} X_{1} & \rightarrow X_{01}
\end{aligned}
$$

are cofibrations in $\mathcal{C}$. (Note that this implies that $A_{01} \rightarrow X_{01}$ is a cofibration too.)

The category $P \mathcal{C}$ will serve as a path object (i.e., a dual cylinder) in CofCat. The cofibrations of $P \mathcal{C}$ are similar but not quite the same as Reedy cofibrations. This modification is important since otherwise the diagonal functor in the proof of Theorem 2.8 below would not be exact.

Proposition 2.7. If $\mathcal{C}$ is a cofibration category, then so is $P \mathcal{C}$ with the above weak equivalences and cofibrations.

Proof.

(C0) Weak equivalences satisfy 2-out-of-6 since this holds in $\mathcal{C}$.

(C1) A morphism $A \rightarrow X$ is an acyclic cofibration if and only if all

$$
\begin{aligned}
A_{0} & \rightarrow X_{0}, \\
A_{1} & \rightarrow X_{1}, \\
A_{01} \amalg_{A_{0}} X_{0} & \rightarrow X_{01} \text { and } \\
A_{01} \amalg_{A_{1}} X_{1} & \rightarrow X_{01}
\end{aligned}
$$

are acyclic cofibrations in $\mathcal{C}$. Hence every isomorphism is an acyclic cofibration.

$(\mathrm{C} 2-3)$ The constant diagram of initial objects is cofibrant and initial in $P \mathcal{C}$. Moreover, the definition of a cofibrant object $X$ is equivalent to $0 \rightarrow X$ being a cofibration, thus all objects of $P \mathcal{C}$ are cofibrant.

(C4) A cofibration in $P \mathcal{C}$ is, in particular, a levelwise cofibration and so pushouts along cofibrations in $P \mathcal{C}$ exist and are constructed levelwise. Given a pushout square,

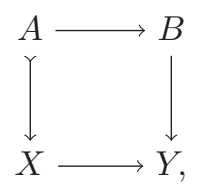

in $P \mathcal{C}$ we observe that $B_{0} \rightarrow Y_{0}$ and $B_{1} \rightarrow Y_{1}$ are pushouts of $A_{0} \rightarrow X_{0}$ and $A_{1} \rightarrow X_{1}$ so they are cofibrations. The Pushout Lemma says that

$$
B_{01} \amalg_{B_{0}} Y_{0} \rightarrow Y_{01} \text { and } B_{01} \amalg_{B_{1}} Y_{1} \rightarrow Y_{01}
$$

are pushouts of

$$
A_{01} \amalg_{A_{0}} X_{0} \rightarrow X_{01} \text { and } A_{01} \amalg_{A_{1}} X_{1} \rightarrow X_{01}
$$


so they are cofibrations too. Consequently, $B \rightarrow Y$ is a cofibration in $P \mathcal{C}$. Stability of acyclic cofibrations under pushouts is obtained by combining this argument with the characterization of acyclic cofibrations given in (C1) above.

(C5) Let $X \rightarrow Y$ be a morphism of $P \mathcal{C}$. For $i \in\{0,1\}$ factor $X_{i} \rightarrow Y_{i}$ as $X_{i} \longmapsto$ $Z_{i} \stackrel{\sim}{\rightarrow} Y_{i}$ in $\mathcal{C}$ and form pushouts

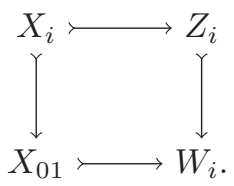

Then we have the induced morphisms $W_{i} \rightarrow Y_{01}$ which make the square

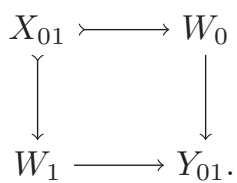

commute and thus yield a morphism $W_{0} \amalg_{X_{01}} W_{1} \rightarrow Y_{01}$. We factor it in $\mathcal{C}$ as

$$
W_{0} \amalg_{X_{01}} W_{1} \longmapsto Z_{01} \stackrel{\sim}{\rightarrow} Y_{01} .
$$

Then $Z$ becomes an object of $P \mathcal{C}$ and $X \longmapsto Z \stackrel{\sim}{\rightarrow} Y$ is a factorization of the original morphism.

We are ready to prove our main result.

Theorem 2.8. The category CofCat with weak equivalences and fibrations as above is a complete fibration category.

In other words CofCat satisfies the duals of axioms $(\mathrm{C} 0-6)$ and $(\mathrm{C} 7-\kappa)$ for all $\kappa$.

Proof.

$(\mathrm{C} 0)^{\text {op }}$ Weak equivalences satisfy 2-out-of-6 since they are created from equivalences of categories by Ho: CofCat $\rightarrow$ Cat.

$(\mathrm{C} 1)^{\text {op }}$ Isomorphisms are acyclic fibrations by Proposition 2.5.

$(\mathrm{C} 2-3)^{\text {op }}$ The category [0] has a unique structure of a cofibration category and it is a terminal cofibration category. Moreover, every cofibration category is fibrant since every category is isofibrant while the lifting properties for factorizations and pseudofactorizations follow from the factorization axiom.

(C4) ${ }^{\text {op }}$ Proposition 2.4 says that pullbacks along fibrations exist and by the construction they are also pullbacks in CofCat. Since fibrations are defined by the right lifting property in this category they are stable under pullbacks. This argument also applies to acyclic fibrations by Proposition 2.5 since (App1) is equivalent to the right lifting property with respect to the inclusion $[1] \hookrightarrow \widehat{[1]}$. 
$(\mathrm{C} 5)^{\mathrm{op}}$ To verify the factorization axiom it suffices to construct a path object for every cofibration category $\mathcal{C}$ by [Bro73, Factorization lemma, p. 421]. Let diag: $\mathcal{C} \rightarrow P \mathcal{C}$ be the diagonal functor. It preserves (acyclic) cofibrations since if $X \longmapsto Y$ is an (acyclic) cofibration in $\mathcal{C}$, then both $(\operatorname{diag} X)_{0} \rightarrow$ $(\operatorname{diag} Y)_{0}$ and $(\operatorname{diag} X)_{1} \rightarrow(\operatorname{diag} Y)_{1}$ coincide with $X \longmapsto Y$ while

$$
(\operatorname{diag} X)_{01} \amalg_{(\operatorname{diag} X)_{0}}(\operatorname{diag} Y)_{0} \rightarrow(\operatorname{diag} Y)_{01}
$$

and

$$
(\operatorname{diag} X)_{01} \amalg_{(\operatorname{diag} X)_{1}}(\operatorname{diag} Y)_{1} \rightarrow(\operatorname{diag} Y)_{01}
$$

are isomorphisms. It also preserves the pushouts along cofibrations and hence is exact. The evaluation functor

$$
\mathrm{ev}_{0,1}=\left(\mathrm{ev}_{0}, \mathrm{ev}_{1}\right): P \mathcal{C} \rightarrow \mathcal{C} \times \mathcal{C}
$$

is also exact. Together they form a factorization of the diagonal functor $\mathcal{C} \rightarrow \mathcal{C} \times \mathcal{C}$. We need to show that diag is a weak equivalence and that $e_{0,1}$ is a fibration.

Consider the evaluation functor $\mathrm{ev}_{01}: P \mathcal{C} \rightarrow \mathcal{C}$. It is a homotopical functor such that $\mathrm{ev}_{01} \operatorname{diag}=\mathrm{id}_{\mathcal{C}}$ and there is a natural weak equivalence $\operatorname{id}_{P \mathcal{C}} \rightarrow$ $\operatorname{diag} \mathrm{ev}_{01}$ since all morphisms of $\widehat{\lrcorner}$ are weak equivalences. It follows that Ho diag is an equivalence.

It is easy to see that $\mathrm{ev}_{0,1}$ is an isofibration. The lifting property for factorizations is verified just like the factorization axiom in $P \mathcal{C}$ except that now the factorizations $X_{i} \longmapsto Z_{i} \stackrel{\sim}{\rightarrow} Y_{i}$ are given in advance. The lifting property for pseudofactorizations is handled similarly: let $X \rightarrow Y$ be a morphism in $P \mathcal{C}$ and let

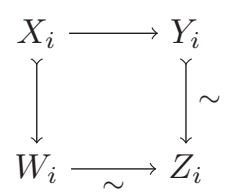

be pseudofactorizations of $X_{i} \rightarrow Y_{i}$ for $i \in\{0,1\}$. Form pushouts
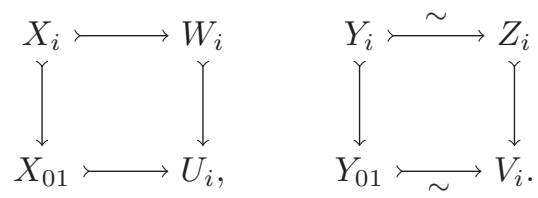

There are induced morphisms $U_{i} \rightarrow V_{i}$ which fit into a commutative diagram

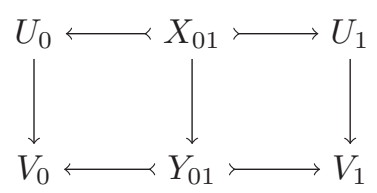

and thus induce a morphism $U_{0} \amalg_{X_{01}} U_{1} \rightarrow V_{0} \amalg_{Y_{01}} V_{1}$ which we pseudofactorize into 


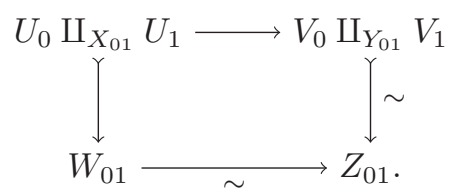

Then $W$ and $Z$ form objects of $P \mathcal{C}$ which fit into a pseudofactorization

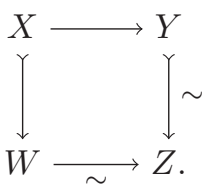

as required.

(C6 $)^{\text {op }}$ The limit of a tower of fibrations between cofibration categories can be constructed as the limit of their underlying categories. The resulting category satisfies the axioms of a cofibration category since the required colimits and factorizations can be constructed by finding suitable lifts along the fibrations of the tower in analogy to Proposition 2.4.

$(\mathrm{C} 7)^{\text {op }}$ The product of a family of cofibration categories can be constructed as the product of their underlying categories. It inherits a structure of a cofibration category by a direct verification.

A routine modification of this argument also yields the following.

Theorem 2.9. For every regular cardinal $\kappa$, the category CofCat ${ }_{\kappa}$ with weak equivalences and fibrations as above is a complete fibration category.

\section{References}

[AG97] K. Andersen and J. Grodal, A Baues Fibration Category Structure on Banach and $C^{*}$-algebras (1997), available at http://www . math.ku.dk/ jg/papers/fibcat.pdf.

[And78] D.W. Anderson, Fibrations and geometric realizations, Bull. Amer. Math. Soc. 84 (1978), no. 5, 765-788.

[AKL15] J. Avigad, K. Kapulkin, and P.L. Lumsdaine, Homotopy limits in type theory, Math. Structures Comput. Sci. 25 (2015), no. 5, 1040-1070.

[Bau89] H.J. Baues, Algebraic homotopy, Cambridge Stud. Adv. Math., vol. 15, Cambridge University Press, Cambridge, 1989.

[Bau99] H.-J. Baues, Combinatorial foundation of homology and homotopy, Springer Monogr. Math., Springer-Verlag, Berlin, 1999.

[Bro73] K.S. Brown, Abstract homotopy theory and generalized sheaf cohomology, Trans. Amer. Math. Soc. 186 (1973), 419-458.

[Cis10a] D.-C. Cisinski, Catégories dérivables, Bull. Soc. Math. France 138 (2010), no. 3, 317-393.

[Cis10b] D.-C. Cisinski, Invariance de la K-théorie par équivalences dérivées, J. K-Theory 6 (2010), no. 3, 505-546. 
[DHKS04] W.G. Dwyer, P.S. Hirschhorn, D.M. Kan, and J.H. Smith, Homotopy limit functors on model categories and homotopical categories, Math. Surveys Monogr., vol. 113, American Mathematical Society, Providence, RI, 2004.

[GZ67] P. Gabriel and M. Zisman, Calculus of fractions and homotopy theory, Ergeb. Math. Grenzgeb., Band 35, Springer-Verlag New York, Inc., New York, 1967.

[Hov99] M. Hovey, Model categories, Math. Surveys Monogr., vol. 63, American Mathematical Society, Providence, RI, 1999.

[KS16] K. Kapulkin and K. Szumiło, Quasicategories of Frames of Cofibration Categories, Appl. Categ. Structures (2016), 1-25.

[RB06] A. Rădulescu-Banu, Cofibrations in Homotopy Theory (2006), available at http://arxiv.org/abs/math/0610009v4.

[Sch84] C. Schochet, Topological methods for $C^{*}$-algebras. III. Axiomatic homology, Pacific J. Math. 114 (1984), no. 2, 399-445.

[Szu14a] K. Szumiło, Two Models for the Homotopy Theory of Cocomplete Homotopy Theories, Ph.D. Thesis, Rheinische Friedrich-Wilhelms-Universität Bonn, 2014, http://hss.ulb.uni-bonn.de/2014/3692/3692.htm.

[Szu14b] K. Szumiło, Two Models for the Homotopy Theory of Cocomplete Homotopy Theories (2014), available at http://arxiv.org/abs/1411.0303.

[Szu16] K. Szumiło, Frames in Cofibration Categories, J. Homotopy Relat. Struct. (2016).

[Szu] K. Szumiło, Homotopy Theory of Cocomplete Quasicategories, To appear in Algebr. Geom. Topol.

[Wal85] F. Waldhausen, Algebraic K-theory of spaces, Algebraic and geometric topology (New Brunswick, NJ, 1983), Lecture Notes in Math., vol. 1126, Springer, Berlin, 1985, pp. 318-419.

Karol Szumiło kszumilo@uwo.ca

Department of Mathematics, University of Western Ontario, 1151 Richmond Street, London, Ontario, N6A 3K7, Canada 